\title{
Identification and Purification of Two Precursors of the Insect Neuropeptide Adipokinetic Hormone
}

\author{
Siegfried Hekimi and Michael O'Shea \\ Université de Genève, Laboratoire de Neurobiologie, Ecole de Médecine, CH-1211 Genève 4, Switzerland
}

\begin{abstract}
Our objective was to establish a system for the investigation of neuropeptide biosynthesis in an insect. To achieve this we developed an in vitro organ culture system for the corpora cardiaca (CC) of the locust (Schistocerca gregaria). The CC are the neurosecretory structures containing the adipokinetic hormones AKH I and AKH II. Tritiated amino acids were added to the glands, and we studied the development of label in newly made proteins using size-exclusion and reverse-phase liquid chromatography. We performed pulsechase and pulse-translation block experiments using ${ }^{3} \mathrm{H}$ tryptophan as tracer. We also raised an antiserum to a synthetic AKH analog and used this in combination with liquid chromatography to identify 2 precursor polypeptides, P1 and P2.
\end{abstract}

Size-exclusion chromatography indicated the presence of a major component of the $\mathrm{CC}$ of about $8 \mathrm{kDa}$ that incorporates ${ }^{3} \mathrm{H}$-tryptophan before AKH I and II. Moreover, in both pulse-chase and pulse-translation block experiments we showed that label is transferred from this $8 \mathrm{kDa}$ component into AKH I. We call this component proAKH; it is recognized by anti-AKH serum in a radioimmunoassay (RIA). Further fractionation of tritium-labeled proAKH by reverse-phase chromatography yielded 2 polypeptides, P1 and P2. Both are AKH-immunoreactive and contain ${ }^{3} \mathrm{H}$-tryptophan after in vitro pulse labeling, and both are proposed precursors of AKH I. The in vitro system we have developed may be a model system for the study of the processes of neuropeptide biosynthesis and its regulation in an intact neurosecretory tissue of an insect.

Most neural tissues are highly heterogenous and the cells synthesizing a particular peptide represent only a small proportion of the total cell population. For this reason, tumor tissue and cell lines have frequently been used as simplified model systems for the study of peptide biosynthesis. The tissue described here is the glandular lobe of the corpus cardiacum (CC), the major neurosecretory structure of insects, which contains adipokinetic hormones (AKHs). It has the advantage of consisting of a relatively homogenous population of peptide-synthesizing neu-

\footnotetext{
Received Oct. 13, 1986; revised Feb. 23, 1987; accepted Apr. 10, 1987.

We thank M. Friedli for expert technical assistance and Tom Barkas for advice in the design of the AKH immunogen. J. Lougheed kindly provided the CC section shown in Figure 1. Drs. J. L. Bény, M. F. Schulz, and K. Siegert reviewed the manuscript, which was prepared with care by E. Jeantin. This work was supported by the Swiss National Fund (Grant 3.181.0-85).

Correspondence should be addressed to Michael O'Shea, Laboratoire de Neurobiologie, Ecole de Médecine, 20 rue de l'Ecole-de-Médecine, CH-1211 Genève 4, Switzerland.

Copyright (C) 1987 Society for Neuroscience $0270-6474 / 87 / 092773-12 \$ 02.00 / 0$
}

rosecretory cells. It has therefore allowed us to develop an in vitro system for the study of neuropeptide synthesis in an intact tissue. In this paper we describe our investigations into the biosynthesis of adipokinetic hormones. They represent the first documentation of neuropeptide synthesis in an insect system.

Adipokinetic hormone (AKH) was the name given (Mayer and Candy, 1969) to a small peptide that causes the mobilization of lipid metabolism, which occurs a few minutes after the initiation of flight in the locust Schistocerca gregaria. The adipokinetic bioactivity is contained in and released from a pair of neurosecretory structures in the head, the corpora cardiaca (CC) (Fig. 1). The CC are connected to the brain and, as their name indicates, are closely associated with the circulatory system, into which they release hormones. Each $\mathrm{CC}$ in the locust consists of 2 lobes, one of which, the glandular lobe, contains neurosecretory cell bodies and their terminals, while the other, the storage lobe, consists of the axon terminals of neurosecretory cells that reside in the brain. In 1976, the complete primary structure of AKH was reported (Stone et al., 1976). The hormone is a blocked decapeptide of the sequence pGlu-Leu-Asn-Phe-Thr-Pro-AsnTrp-Gly-Thr- $\mathrm{NH}_{2}$. The CC are an extremely rich source of this peptide, which can be found in quantities as high as $1 \mathrm{nmol} /$ CC (wet weight, $0.1 \mathrm{mg}$ ). Subsequent to the characterization of AKH, a second peptide with adipokinctic activity was purificd from the CC of the same species $S$. gregaria. This peptide, AKH II, was partly characterized by Carlsen et al. (1979) and was recently sequenced (Siegert et al., 1985). The second adipokinetic hormone, AKH II, is a blocked octapeptide, homologous to the AKH decapeptide (henceforth referred to as AKH I). The sequence of AKH II is pGlu-Leu-Asn-Phe-Ser-Thr-Gly-Trp$\mathrm{NH}_{2}$. The $\mathrm{CC}$ are also a rich source of AKH II $(\sim 200 \mathrm{pmol} /$ $\mathrm{CC}$ in adults). The AKHs are members of a large and growing family of arthropod neuropeptides (O'Shea and Schaffer, 1985). Synthetic AKH I and II are both available (Peninsula Labs) and are chromatographically and biologically indistinguishable from the native hormones.

Reverse-phase and size-exclusion chromatography of total glandular lobe extract (see Results and Siegert and Mordue, 1986) indicated that AKH I and II are the primary products of the intrinsic neurosecretory cells of locust $\mathrm{CC}$. This suggested that the $\mathrm{CC}$ would provide a simple model system for the study of insect neuropeptide synthesis in an intact tissue. For our studies we have developed an in vitro system for short-term culture of the locust CC. This has permitted us to study AKH synthesis by following the incorporation of tritium-labeled amino acids. We report here that the glandular lobes of the $\mathrm{CC}$ are indeed the sites of synthesis for AKH I and II. In addition, we have identified and purified 2 polypeptides (P1 and P2) that are 


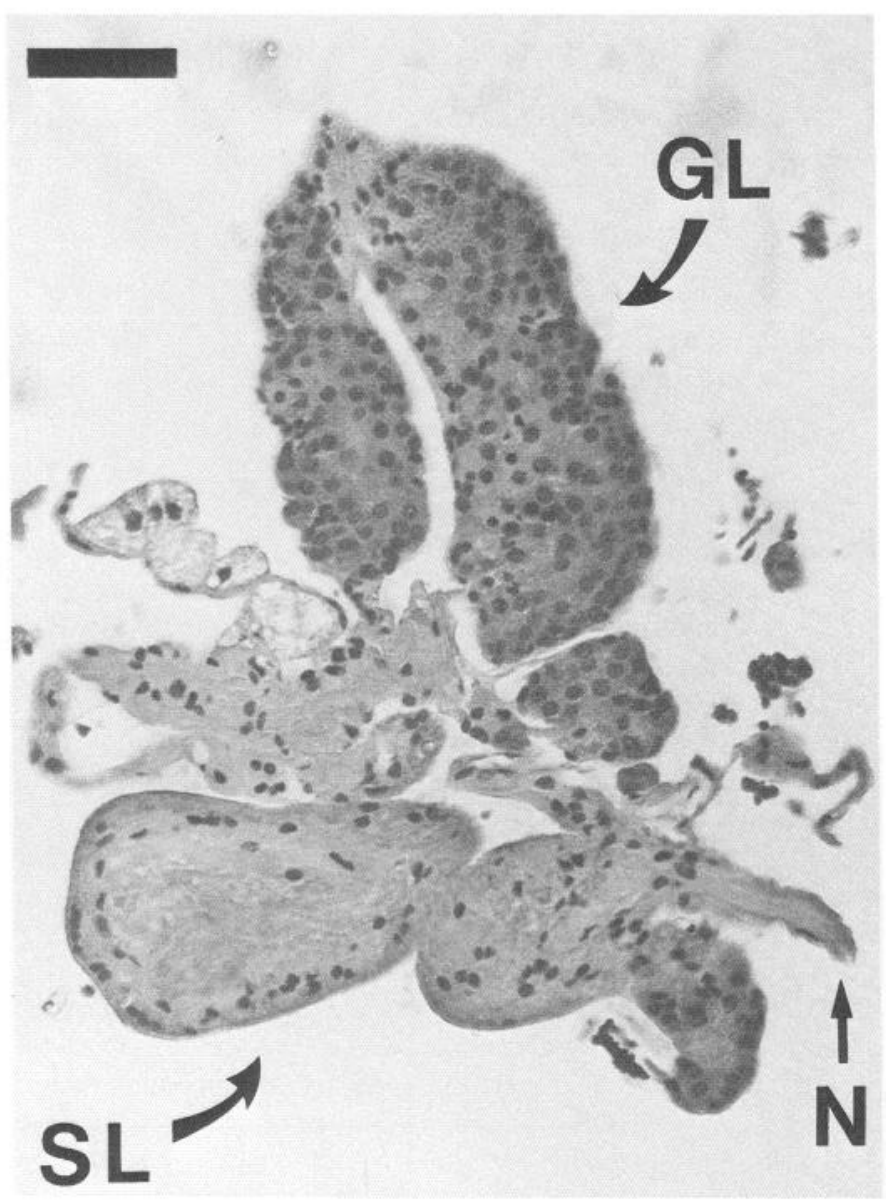

Figure 1. Micrograph of a frontal section through the CC, stained with Mayer's hemalum-eosin. Notice the paired glandular lobes $(G L)$ and storage lobes $(S L)$. One of the nerves connecting the $\mathrm{CC}$ to the brain is also shown $(N)$. Scale, $10 \mu \mathrm{m}$.

synthesized in the glandular lobes. We present several lines of experimental evidence showing that $\mathrm{P} 1$ and $\mathrm{P} 2$ are precursors of AKH I. These include pulse-chase and pulse-translation block experiments and the use of an anti-AKH serum to recognize AKH-immunoreactive polypeptides. This represents the first identification of antecedents for an insect neuropeptide and establishes that neuropeptides in insects are synthesized, as shown in other organisms, from larger precursor polypeptides (Douglass et al., 1984).

\section{Materials and Methods}

Animals. Both male and female adult specimens of the locust Schistocerca gregaria were used in our experiments. They were reared in our own laboratory culture. Female New Zealand White rabbits produced the antisera to a synthetic analog of AKH I.

Preparation of $A K H$ antiserum and radioimmunoassay procedures. To generate an antiserum to the precursor form of AKH I, an analog of the sequence Lys-Tyr-Leu-Asn-Phe-Thr-Pro-Asn-Trp-Gly-Thr-OH was designed and synthesized (Peninsula Laboratories). This peptide is a nonamidated analog of the complete AKH I sequence without pGlu and with Lys-Tyr at the amino terminal. Since it includes almost the entire AKH I sequence and therefore overlaps the AKH II sequence, immunization is likely to generate antisera that recognize both AKH I and AKH II. The terminal lysine residue was provided for efficient cross-linking, and the tyrosine for convenient radiolabeling. The peptide was linked to keyhole limpet hemocyanin $(\mathrm{KLH})$ with glutaraldehyde, emulsified with Freund's adjuvant, and injected subcutaneously into female New Zealand White rabbits. A second injection was made 3 weeks later. Serum was first tested for the presence of $\mathrm{AKH}$ antibodies one week after the second injection, using a radioimmunoassay (RIA). For the RIA, the synthetic AKH I analog was radiolabeled on the tyrosine residue with ${ }^{125}$ I. Radioiodination was carried out using the chloramine $\mathrm{T}$ method.

Activated charcoal was used to precipitate unbound peptide after the serum (diluted 1500 -fold) was incubated with the radiolabeled tracer peptide and samples. The ability of unlabeled synthetic AKH I and II to displace label was measured by counting a $200 \mu \mathrm{l}$ aliquot of the supernatant in a $\gamma$-scintillation counter. A similar procedure was used in testing the $\mathrm{AKH}$-like immunoreactivity of tissue extracts fractionated by high-pressure liquid chromatography (HPLC). In this case the ability of the HPLC fractions to displace bound counts was determined. Details of HPLC procedures are provided below.

In vitro organ culture and $A K H$ synthesis. Living $C C$, including both glandular and storage lobes, were dissected from adult $S$. gregaria and placed into a $100 \mu \mathrm{l}$ drop of insect saline in a petri dish. The saline composition was as follows: $140 \mathrm{~mm} \mathrm{NaCl} ; 5 \mathrm{~mm} \mathrm{KCl} ; 5 \mathrm{~mm} \mathrm{CaCl} ; 1$ $\mathrm{mm} \mathrm{MgCl}_{2} ; 4 \mathrm{mM} \mathrm{NaNCO}_{3} ; 5 \mathrm{~mm}$ trehalose; $5 \mathrm{~mm} \mathrm{~N}$-Tris-OH-methyl2-aminoethanesulfonic acid; $100 \mathrm{~mm}$ sucrose; $\mathrm{pH}$ 7.2. A $100 \mu \mathrm{l}$ drop could accommodate up to $10 \mathrm{CC}$. All incubations were carried out in a humidified chamber at $28^{\circ} \mathrm{C}$. In order to monitor AKH I synthesis, $10 \mu \mathrm{Ci}$ of ${ }^{3} \mathrm{H}$ [side chain 2,3 ] L-tryptophan $(60 \mathrm{Ci} / \mathrm{mmol})$ was added to the saline. This amino acid was selected after testing each of the amino acids present in the AKH sequences. Tryptophan is incorporated with the highest efficiency. This may reflect the relative rarity of tryptophan in the pool of amino acids available to the gland for peptide synthesis. Following varying durations of incubation (see Results) with labeled tryptophan, the CC were either washed in physiological saline without ${ }^{3} \mathrm{H}$-tryptophan and sonicated (see below) or transferred to a large volume $(5 \mathrm{ml})$ of either a "cold-chase" or "translation-block" medium. The cold-chase medium consisted of saline containing $2 \mathrm{~mm}$ of unlabeled tryptophan. The translation-block medium consisted of saline containing $50 \mu \mathrm{g} / \mathrm{ml}$ cycloheximide. During these experiments the glands were placed periodically in fresh media to aid in washing out the unincorporated label.

While entire glands (storage plus glandular lobes) were incubated, the glandular and storage lobes were separated prior to extraction and subjected to separate HPLC analysis. Biosynthetically labeled and unlabeled compounds were identified and purified as described below.

Extraction, identification, and purification procedures. Corpora cardiaca were homogenized in $100 \mu \mathrm{l}$ of $0.1 \%$ trifluoroacetic acid (TFA) at room temperature with a Branson microprobe ultrasound cell disruptor. The CC tissue is small (see Fig. 1) and delicate, and is completely disrupted and dispersed by a very brief $(2-5 \mathrm{sec})$ exposure to ultrasound. The sonication proceeded for $15 \mathrm{sec}$. The homogenate was then centrifuged to eliminate insoluble debris. The crude TFA-soluble supernatant was removed, frozen immediately, and subjected to 2 types of liquid chromatography, size-exclusion gel-permeation and reverse-phase chromatography.

For size fractionation, a $60 \mathrm{~cm}$ TSK $2000 \mathrm{SW}$ gel-permeation column (LKB), eluted at $0.5 \mathrm{ml} / \mathrm{min}$ with $0.1 \mathrm{M} \mathrm{PO}_{4}$ buffer $(\mathrm{pH} 7.0$ ) containing $15 \%$ (vol/vol) acetonitrile, was used. The exclusion size of this column is about $50 \mathrm{kDa}$. Acetonitrile was used to prevent hydrophobic interactions, which result in anomalous retentions of some peptides and tryptophan. Peaks from the size-exclusion column were further purified and identified by rechromatography, using different types of reversephase chromatography (see below). The TSK 2000 column was calibrated according to molecular weight, using the following standards: ferritin, $445 \mathrm{kDa}$ (to determine exclusion volume); trypsin, $24 \mathrm{kDa}$; lysozyme, $14.4 \mathrm{kDa}$; aprotinin, $6.5 \mathrm{kDa}$; ACTH, $4.5 \mathrm{kDa}$; AKH I, 1.1 $\mathrm{kDa}$. The calibration curve shows good linearity and a high correlation $(r=0.96)$ between retention and molecular weight.

Two types of reverse-phase columns were used. Separations of AKH I from AKH II were achieved in 20 min using a Waters $\mu$-Bondapak $\mathrm{C}_{18}$ column eluted at $1 \mathrm{ml} / \mathrm{min}$ with $50 \mathrm{~mm}$ ammonium acetate and a linear acetonitrile gradient (from 20 to $40 \%$ ). Retention times of synthetic AKH I or II (Peninsula Labs) could not be distinguished from those of native unlabeled and native biosynthetically ${ }^{3} \mathrm{H}$-labeled $\mathrm{AKH}$ I or II. The identity of the AKH peptides was further confirmed using additional HPLC systems and eluting conditions. The $C_{18}$ column was isocratically eluted with a sodium phosphate buffer $(0.1 \mathrm{M}, \mathrm{pH} 7.0)$ with $28 \%$ acetonitrile, as well as with a linear gradient of acetonitrile (20$40 \%$ at $1 \% / \mathrm{min}$ ).

We also eluted the $\mathrm{C}_{18}$ column in $0.1 \%$ TFA with either a constant 


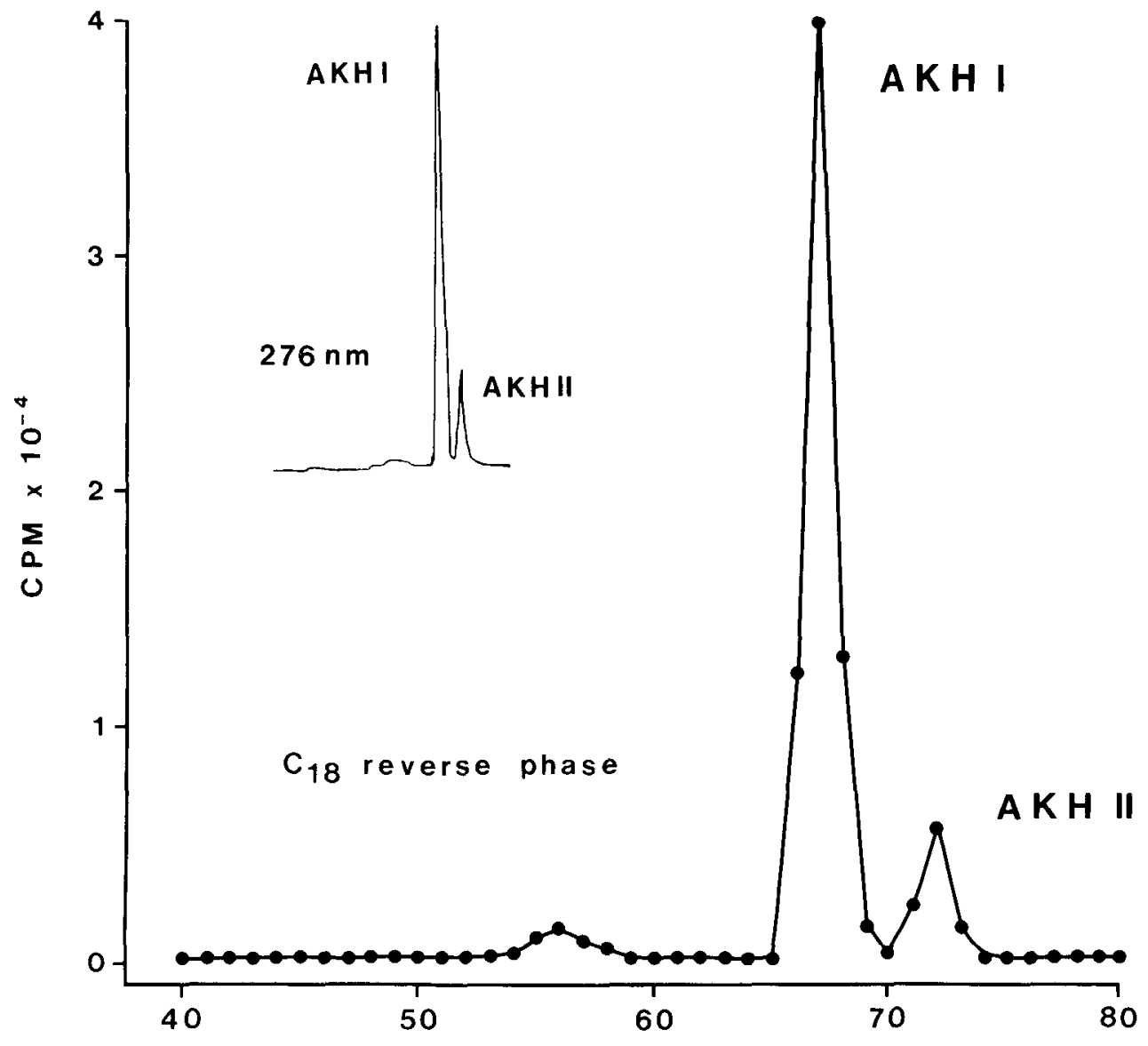

FRACTIONS
Figure 2, Reverse-phase $\left(\mathrm{C}_{18}\right)$ separation of $\mathrm{AKH} \mathrm{I}$ and $\mathrm{AKH}$ II. The inset, upper left, shows the fluorometric trace of an extract of 10 adult male CC. The fluorescence ratio of $0.2(\Lambda \mathrm{KH} \mathrm{II} / \mathrm{\Lambda KH}$ I) is typical for adult males. The graph ( $200 \mu 1 /$ fraction) shows the results of an incubation of 10 adult male $\mathrm{CC}$ with ${ }^{3} \mathrm{H}$-tryptophan for $24 \mathrm{hr}$. The isotope is found in both $\mathrm{AKH}$ I and AKH II and the ratio of labeled peptides is similar to the ratio of fluorescence. Since each AKH peptide contains only a single tryptophan, the $276 \mathrm{~nm}$ fluorescence is a measure of the relative amounts of the 2 . Incorporation rates, therefore, reflect the steady state levels of the peptides. concentration $(28 \%)$ or a linear gradient $(20-40 \%, 1 \% / \mathrm{min})$ of acetonitrile. In addition, the biosynthetically labeled and unlabeled hormones were isolated by $\mathrm{C}_{8}$ reverse-phase chromatography (Protesil $300 \AA$ Å octyl; Whatman), using the same variations of eluting conditions described above for the $\mathrm{C}_{18}$ column. No conditions could be found that separated native (unlabeled or biosynthetically labeled) from synthetic AKH I and II. Thus we are confident that the purified native material and the synthetic standards are the same.

The $\mathrm{C}_{18}$ column was not suitable for purification of the AKH I precursors (P1 and P2). Separation and purification of the precursors was achieved using the Whatman Protesil $300 \AA$ octyl $\mathrm{C}_{8}$ reverse-phase column. The column was eluted in $20 \mathrm{~min}$ at $1 \mathrm{ml} / \mathrm{min}$ in $0.1 \%$ TFA with a linear gradient of acetonitrile (from 40 to $60 \%$ ). While attempting to check the homogeneity of $\mathrm{P} 1$ and $\mathrm{P} 2$, we also chromatographed them using different eluting conditions. These included $0.1 \mathrm{M}$ sodium phosphate $(\mathrm{pH} 7.0)$ both isocratically $(50 \%)$ and with various gradients of acetonitrile concentration.

Four types of detection systems, 2 "on-line" and 2 following fractionations, were used to identify the eluting compounds. On-line detection was provided by a Waters 440 fixed-wavelength $(214 \mathrm{~nm})$ absorbance detector, which gave an estimate of the relative abundance of peptide bonds, and by a Kratos fluorometer set at an excitation wavelength of $276 \mathrm{~nm}$ to reveal tryptophan-containing peptides and proteins. In some experiments, compounds were detected by either RIA or scintillation counting after fractionation of the eluant. For detection of the AKH immunoreactivity of the putative precursors, HPLC fractions $(200$ $\mu$ l) were evaporated to dryness, using a Savant Speed-Vac evaporator, and subjected to RIA (see above).

\section{Results}

Site of AKH synthesis

The AKH I and II peptides are major products of the glandular lobes of the corpora cardiaca, being present in amounts ranging from 0.2 to $1 \mathrm{nmol} / \mathrm{CC}(\mathrm{AKH} \mathrm{I})$ and $0.1-0.4 \mathrm{nmol} / \mathrm{CC}(\mathrm{AKH}$ II) in adult animals. Since neither peptide is present in the storage lobe, the most likely site of $\mathrm{AKH}$ synthesis is in the intrinsic neurosecretory cells of the glandular lobe (Fig. 1).

To address the question of the site of synthesis directly, we cultured groups of $\mathrm{CC}$ in vitro in the presence of tritiated tryptophan. We then separately extracted glandular and storage lobes. The extracts were subjected to $\mathrm{C}_{18}$ reverse-phase chromatography, and the incorporation of radiolabel into newly synthesized peptides was measured. No incorporation could be detected in the storage lobe. The results of a glandular lobe analysis are shown in Figure 2. The tritium isotope can be found coeluting with both $\mathrm{AKH}$ peptides and first becomes detectable in the peptides after about $20-30 \mathrm{~min}$ of incubation. The incorporation of ${ }^{3} \mathrm{H}$-tryptophan provides a measure of the relative rates of synthesis of AKH I and II. There was always more incorporation into AKH I than into AKH II. In fact, the ratio of the amounts of tritium incorporation into the 2 peptides at any one time closely matched the ratio of the absolute amounts of the 2 peptides stored in the glands. This can be seen in Figure 2, in which the AKH II:AKH I ratio measured from the 276 $\mathrm{nm}$ fluorescence is about 0.2 (see inset in Fig. 2)-about the same as the ratio of tritium for the 2 peaks and typical for adult animals. Note that since each peptide contains one tryptophan residue, the relative fluorescence of the AKHs is a reliable measure of their relative abundance. Perhaps the simplest interpretation of this result is that the marked difference in the stored amounts of the $2 \mathrm{AKH}$ peptides is achieved through differential 


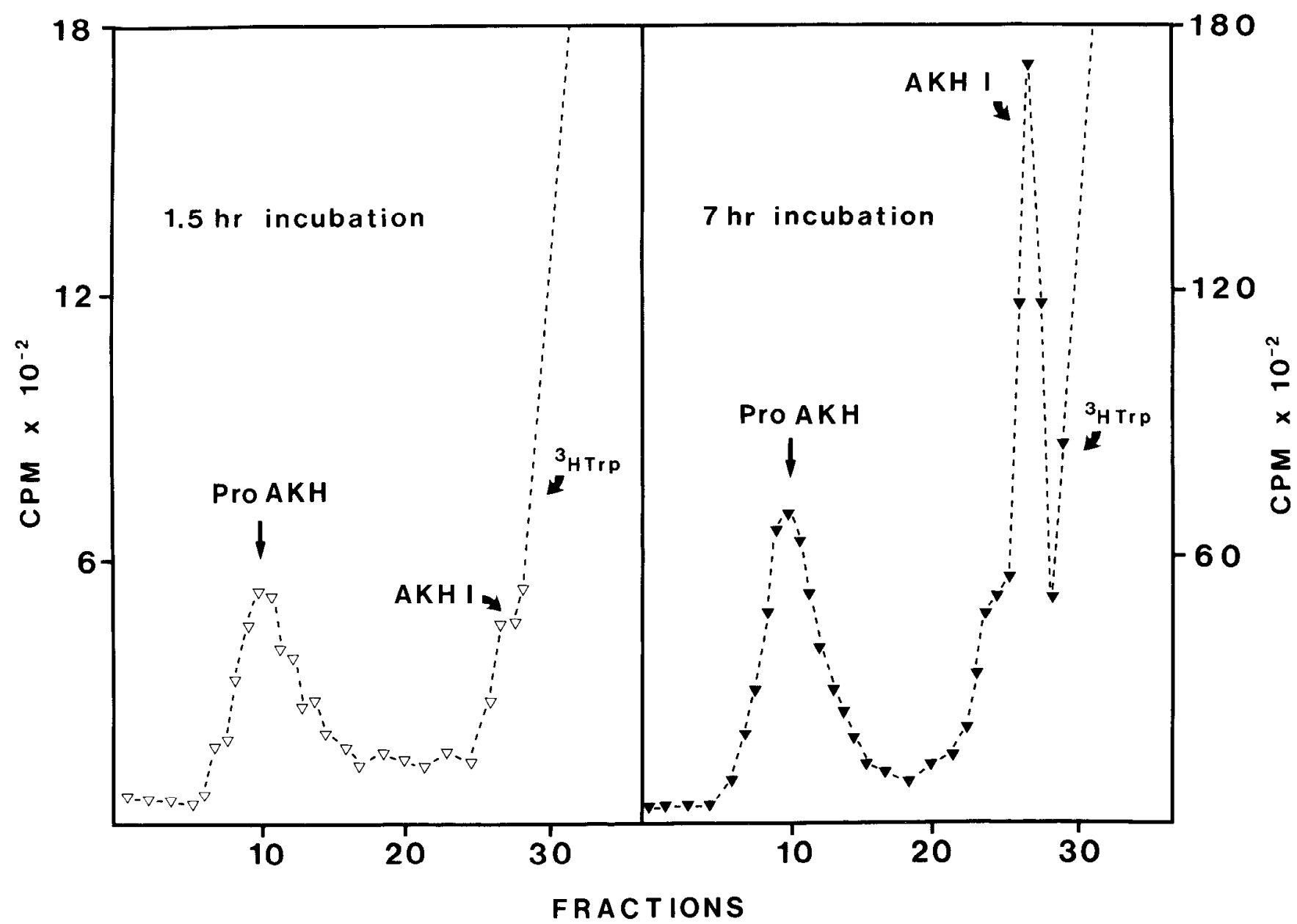

Figure 3. Separations according to molecular size (TSK $2000 \mathrm{SW}$ ) of radiolabeled compounds extracted from CC incubated in the presence of ${ }^{3} \mathrm{H}$-tryptophan for 1.5 and $7 \mathrm{hr}$. Notice that after $1.5 \mathrm{hr}$ of incubation, there is a significant incorporation into a peak (proAKH) near fraction 10 . This indicates, by comparison with standards (see Materials and Methods), a molecular weight of about $8 \mathrm{kDa}$. Few counts appear in AKH I after such a short incubation. After $7 \mathrm{hr}$ there is a major incorporation into AKH I and an approximate 10-fold increase in incorporation into proAKH. Note the difference in the $\mathrm{cpm}$ scales for each. Fractions are $250 \mu \mathrm{l}$. At the end of each chromatographic profile, the free or unincorporated ${ }^{3} \mathrm{H}$ tryptophan elutes and prevents the measurement of label in AKH II, with which it partly comigrates. In the pulse-chase experiments, however (see Fig. 5), the free ${ }^{3} \mathrm{H}$-tryptophan is displaced by the unlabeled, and this allows us to see ${ }^{3} \mathrm{H}-\mathrm{AKH}$ II after incubation.

regulation of their respective rates of synthesis. None of the chromatographic procedures used (see Materials and Methods) could separate biosynthetically labeled AKH I and II from unlabeled native or synthetic peptides.

\section{Identification of AKH precursor (proAKH)}

The in vitro $\mathrm{CC}$ culture system, combined with size-exclusion chromatography, allowed us to identify antecedents of glandular lobe hormones. To accomplish this, we incubated glands in the presence of ${ }^{3} \mathrm{H}$-tryptophan for periods of time too brief to allow the detection by TSK 2000 chromatography of significant levels of radiolabeled AKH I. The incubated glands were extracted and chromatographed by size-exclusion gel-permeation liquid chromatography on a TSK $2000 \mathrm{SW}$ column. This allowed us to identify an $8 \mathrm{kDa}$ peak that fulfilled some of the criteria defining a precursor. For example, such a compound must be made before the adipokinetic hormones, it must be of higher molecular weight, and, of course, it must incorporate ${ }^{3} \mathrm{H}$-tryptophan, an amino acid contained in both AKH I and II.

Figure 3 shows the TSK 2000 chromatograms of a short- (1.5 $\mathrm{hr})$ and a longer- $(7 \mathrm{hr})$ duration in vitro synthesis experiment.
The glands made very little radiolabeled AKH I during the shorter incubation. During this period, however, the tritium isotope was incorporated into a peak eluting with an apparent molecular weight of about $8 \mathrm{kDa}$. In the longer incubation (right, Fig. 3), this $8 \mathrm{kDa}$ peak incorporated more counts, and there was an increased accumulation of isotope comigrating with $\mathrm{AKH}$ I. The tritiated AKH II peak in this experiment is hidden by the large number of counts of unincorporated ${ }^{3} \mathrm{H}$-tryptophan that elute at the end of the chromatogram. The $8 \mathrm{kDa}$-labeled compound comigrates with a major optical density peak shown in Figure 4. In fact, when detecting optical density at $214 \mathrm{~nm}$, the size-exclusion chromatograph of a crude CC extract shows a simple pattern with 2 major peaks. One comigrates with the tritiated proAKH peak illustrated in Figure 3, and the other is AKH I (see Figs. 3, 4). The $8 \mathrm{kDa}$ peak identified as proAKH in Figure 4 is, of course, not necessarily pure, and the optical density peak may not be the labeled peak proAKH in Figure 3. Gel-permeation chromatography is not a high-resolution technique, and comigration, at best, means that compounds of similar molecular weight are not discriminated. At this point in the experiments, however, proAKH is defined as a peak of biosyn- 


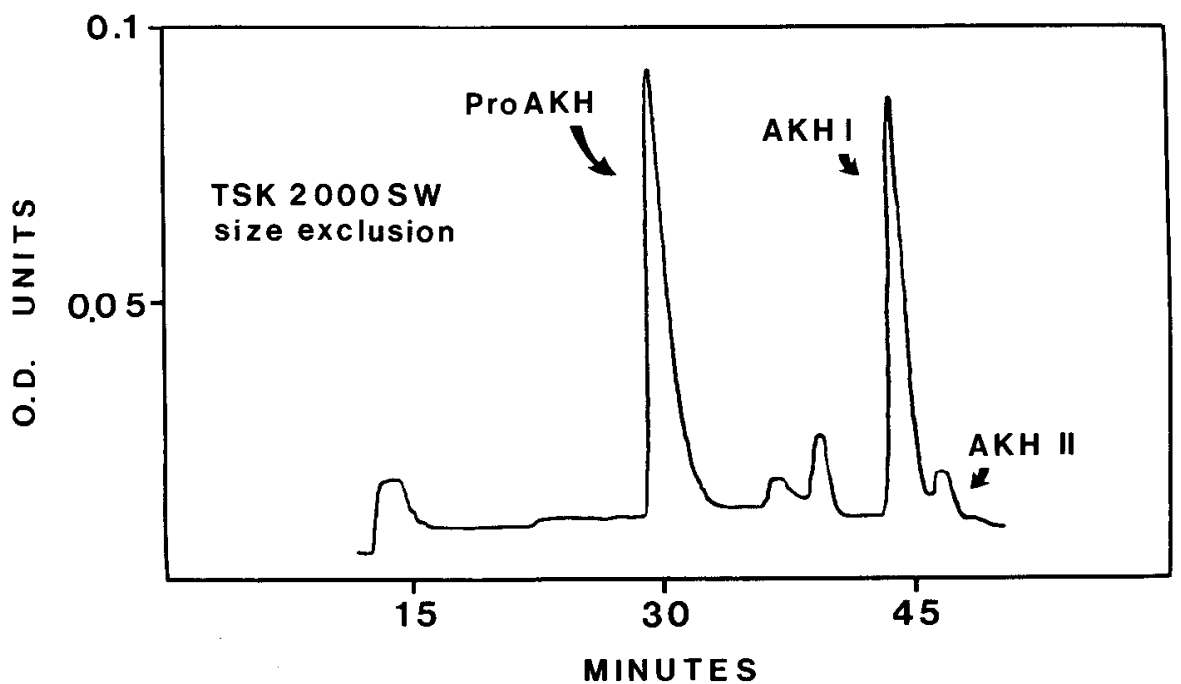

Figure 4. Size-exclusion chromatography of a CC extract ( $3 \mathrm{CC}$ ). Detection is by optical density at $214 \mathrm{~nm}$. The major peak labeled (proAKH) comigrates with biosynthetically labeled ${ }^{3} \mathrm{H}$ proAKH, shown in Figure 3. It has a molecular weight of approximately 8 $\mathrm{kDa}$, as determined by comparison with standards. The only other major optical density peak is AKH I. Notice that the decapeptide (AKH I) and octapeptide (AKH II) are resolved by this chromatographic system. thetically labeled material of about $8 \mathrm{kDa}$; that it comigrates with a major optical density and fluorescence peak may be coincidental. Later, however, we will show that, in fact, biosynthetically labeled proAKH consists primarily of 2 components, which co-elute on the TSK 2000 column.

The identity and purity of the AKH I and II peaks indicated in Figure 4 are confirmed by chromatography on 2 types of reverse-phase columns $\left(\mathrm{C}_{18}\right.$ and $\left.\mathrm{C}_{8}\right)$ and 3 types of elution (see Materials and Methods). Each produces a single peak, which comigrates with synthetic standards of either AKH I or II. That the $\mathrm{AKH}$ peaks identified by size-exclusion chromatography are pure and authentic has also been independently shown quantitatively by Siegert and Mordue (1986).

The time course of the appearance of label in the proAKH peak and in AKH I is shown in Figure 5. During continuous incubation with tritiated tryptophan, the tritium isotope first appears in the $8 \mathrm{kDa}$ compound, but it does not accumulate there indefinitely. The label in this compound reaches a plateau in about $5 \mathrm{hr}$. By contrast, the radiolabel in AKH I appears later and accumulates. The accumulation is initially slow and nonlinear; then, the label in the peptide accumulates linearly. In summary, Figure 5 shows that label in the precursor reaches a maximum in about $5 \mathrm{hr}$ and that, at about the same time, accumulation of label in AKH I becomes linear with respect to the duration of incubation.

\section{Fate of label in precursor}

Two types of experiment, pulse-chase and pulse-translation block, were performed to follow the development of label appearing in proAKH. In the pulse-chase experiments we incubated the glands for $1.5 \mathrm{hr}$ in the presence of ${ }^{3} \mathrm{H}$-tryptophan. When the TSK $2000 \mathrm{SW}$ column is used for fractionation, this is too short a period to produce a significant label in AKH I. After the $1.5 \mathrm{hr}$ pulse, the glands were transferred into the chase medium containing unlabeled tryptophan. A chase duration of about $12 \mathrm{hr}$ was generally sufficient for complete loss of the label in proAKH to occur. Figure 6 is an example of such an experiment, in which the chase duration was $19 \mathrm{hr}$. Label can now be seen comigrating with both AKH I and II. Notice that, at the end of the chromatogram of the $1.5 \mathrm{hr}$ pulsed $\mathrm{CC}$, a large tail of unincorporated labeled tryptophan appears. This comigrates with AKH II and therefore prevents the measurement of tritium in AKH II except after a long-duration wash in the chase medium, as illustrated on the right panel of Figure $6(19 \mathrm{hr}$ chase). Here the unincorporated ${ }^{3} \mathrm{H}$-tryptophan has been washed out and diluted sufficiently to allow a labeled peak comigrating with AKH II to be seen. Confirmation of the identity of the 2 biosynthetically labeled AKH peaks isolated by this size-exclusion chromatography has been achieved by a number of reverse-phase chromatographic systems, as described above. Furthermore, the identity of native AKH I and II isolated by gel-permeation chromatography has been shown by independent investigators (Siegert and Mordue, 1986).

In addition to performing complete chase experiments, we also observed the development of the label at regular intervals during the chase in order to quantify the transfer of counts from the labeled proAKH peaks into AKH I (Fig. 7). For these experiments, groups of 3 glands were incubated for $1.5 \mathrm{hr}$ and

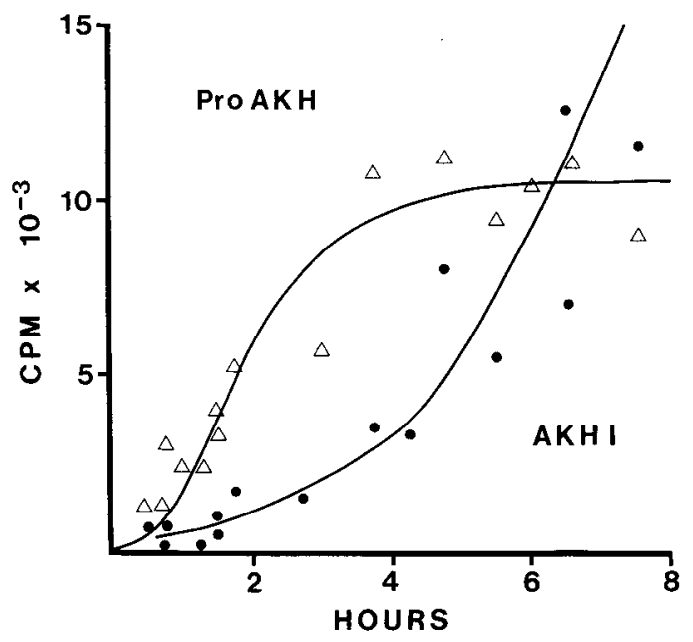

Figure 5. Time course of incorporation of ${ }^{3} \mathrm{H}$-tryptophan into proAKH and AKH I. The data are taken from experiments of the type illustrated in Figure 3, in which radiolabeled products are separated by TSK $2000 \mathrm{SW}$ chromatography following varying durations of incubation in saline containing ${ }^{3} \mathrm{H}$-tryptophan. Each point is the sum of counts found in either proAKH (open triangles) or AKH I (closed circles). Three glandular lobes incubated under the same conditions contribute to each point. Notice that incorporation into proAKH does not increase significantly after $5 \mathrm{hr}$, but that incorporation into AKH I becomes more or less linear at this time. For further discussion, see text. 


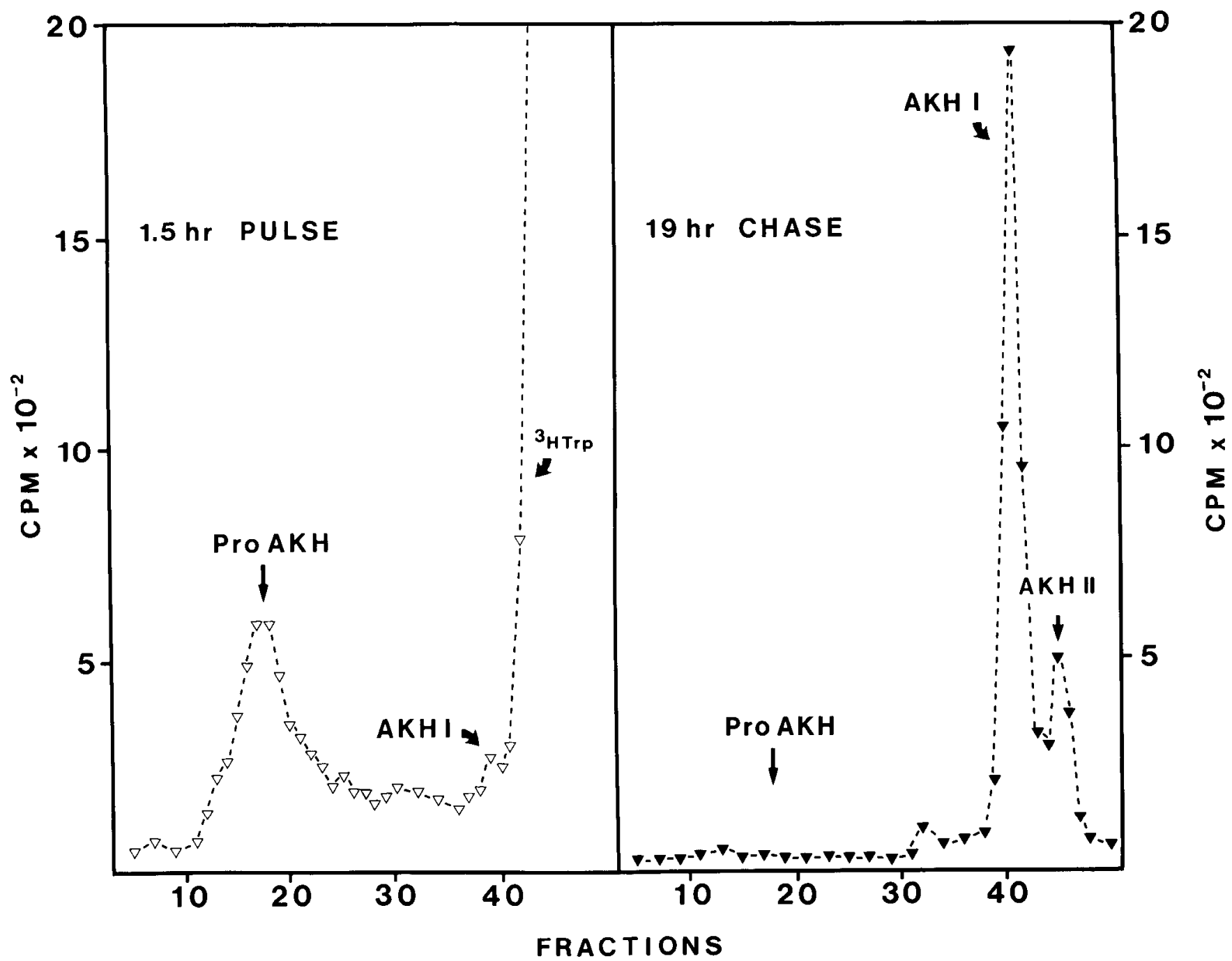

Figure 6. Complete loss of counts from pulse-labeled proAKH and the appearance of label in AKH I and II following a long-duration chase. Notice that the free ${ }^{3} \mathrm{H}$-tryptophan tail is absent after the chase, allowing us to measure the isotope in AKH II (compare to Fig. 3). Separations were performed on the TSK $2000 \mathrm{SW}$ column. Fractions are $250 \mu \mathrm{l}$.

then placed in chase medium for variable periods. Each point in Figure 7 represents the counts (pooled from 3 glands) found in proAKH and AKH I in experiments in which intact glands were pulsed, chased for variable periods, and then extracted and fractionated by size-exclusion chromatography on the TSK 2000 SW column. This time course experiment reveals a close mirror symmetry of the points representing the disappearance of label from proAKH and its appearance in AKH I. Though scatter of data can be expected in experiments of this type on cultured intact tissue, the relationships and symmetry are in fact good, and provide quantitative evidence that label is transferred from proAKH into AKH I in a time-dependent manner. In this experiment, it is not possible to quantitate the time course of the transfer of label into AKH II because unincorporated ${ }^{3} \mathrm{H}$-tryptophan comigrates with the octapeptide on the size-exclusion column (see Fig. 6). Compared to AKH I, however, AKH II is a relatively minor product (about $10-20 \%$ of AKH I levels in adult animals) and we would expect, therefore, that counts lost from proAKH in the pulse-chase experiment would be largely recovered in $\mathrm{AKH} \mathrm{I}$, as is shown in Figure 7. Indeed, since some unincorporated ${ }^{3} \mathrm{H}$-tryptophan is still present in the glands dur- ing the early part of the chase, we expect that some incorporation into proAKH will continue for a while during the chase. This is indicated by the observation that it is sometimes possible to find slightly more counts in AKH I at the end of the chase than were present in proAKH at the end of the pulse. In general, however, considering that intact tissue is being used, the quantitative relationships are good and show that most, if not all, the label incorporated into proAKH during a $1.5 \mathrm{hr}$ pulse is transferred to the AKH peptides within $20 \mathrm{hr}$.

In another series of pulse-labeling experiments, we studied the fate of tritium in proAKH under conditions in which continued protein synthesis was prevented; these are called pulsetranslation block experiments. Glands were incubated for 1.5 hr in the normal pulse medium, containing ${ }^{3} \mathrm{H}$-tryptophan, and then transferred into a medium containing $50 \mu \mathrm{g} / \mathrm{ml}$ cycloheximide. In control experiments, we first determined that in the presence of this concentration of cycloheximide, the appearance of label in proAKH is completely blocked and that no label appears in AKH I. Thus we could assume that in our in vitro culture system, cycloheximide stops translation by the ribosomes in the glandular lobes rapidly enough so that, in its 

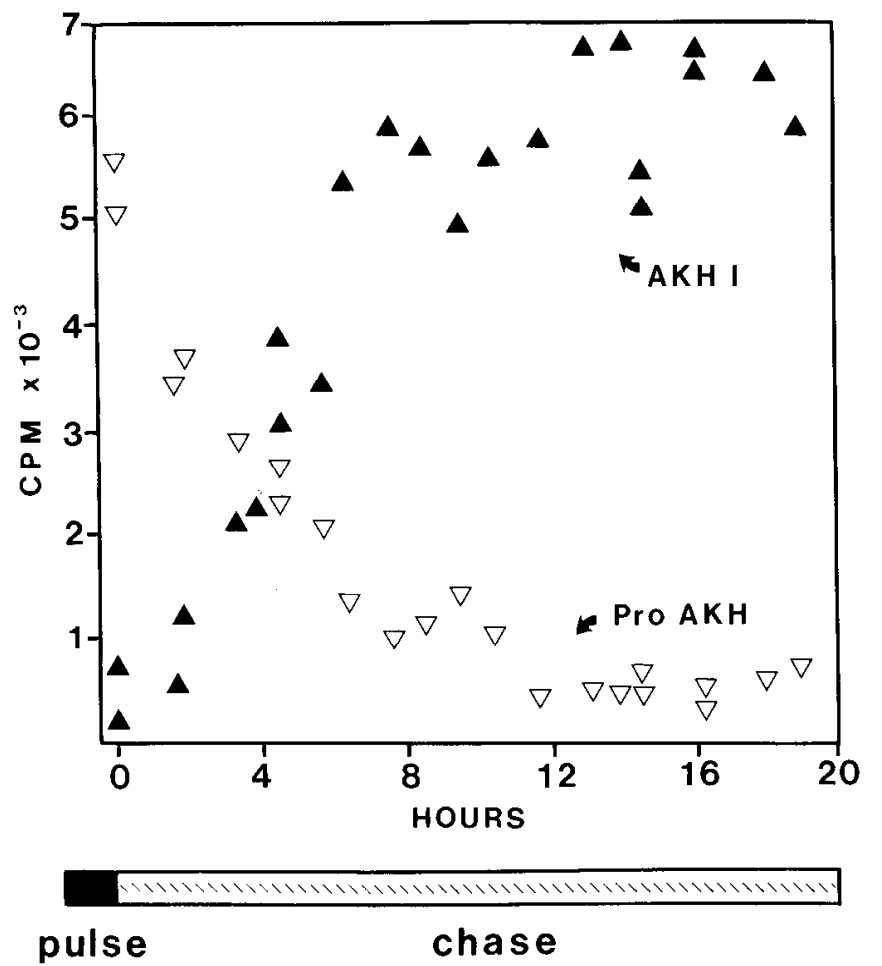

Figure 7. Time course of pulse-chase transfer of counts from proAKH into $\mathrm{AKH} \mathrm{I}$. Following a standard $1.5 \mathrm{hr}$ incubation in the presence of ${ }^{3} \mathrm{H}$-tryptophan ( $p$ ulse), the glands were placed in chase medium. Three glands were removed and extracted at different times during the chase. For each sample of 3 glands, radiolabeled products were separated by TSK $2000 \mathrm{SW}$ chromatography, and counts representing proAKH and AKH I were measured. Notice the close symmetry between the loss of counts in proAKH and the appearance of counts in AKH I. Complete transfer is achieved within $12 \mathrm{hr}$.

presence, we cannot detect synthesis of labeled proAKH. Thus the addition of cycloheximide to glands previously pulsed with tritiated tryptophan prevents further incorporation of label in the precursor. Cycloheximide would not, however, be expected to prevent enzymatic processing of previously synthesized proAKH, and would not, therefore, prevent the transfer of label into AKH. Figure 8 confirms this expectation and shows the time course of the progress of label in proAKH during pulsetranslation block experiments. As during the pulse-chase experiment, label is lost from the precursor and largely recovered in AKH I. In the block experiment, however, there can be no ongoing synthesis, so all the label finally found in AKH I must have been present in the precursor before blocking translation. It can be seen from Figure 6 that after a $1.5 \mathrm{hr}$ pulse, only proAKH has sufficient label to account for the label that subsequently appears in AKH I in either pulse-chase or pulse-translation block experiments. In summary, at this point in our experiments we defined as proAKH a tritiated component with a peak migrating on a TSK $2000 \mathrm{SW}$ gel-permcation column, with an apparent molecular weight of about $8 \mathrm{kDa}$. It rapidly incorporates ${ }^{3} \mathrm{H}$-tryptophan, it does not accumulate, and the isotope in pulse-labeled proAKH is largely transferred into $\mathrm{AKH} \mathrm{I}$, where label accumulates. As we show in Figures 2 and $6,{ }^{3} \mathrm{H}-$ AKH II is also made in our tissue culture experiments, but it represents only a small percentage of the label found in the more abundant AKH I peptide.

Additional evidence linking proAKH to the synthesis of $\mathrm{AKH}$

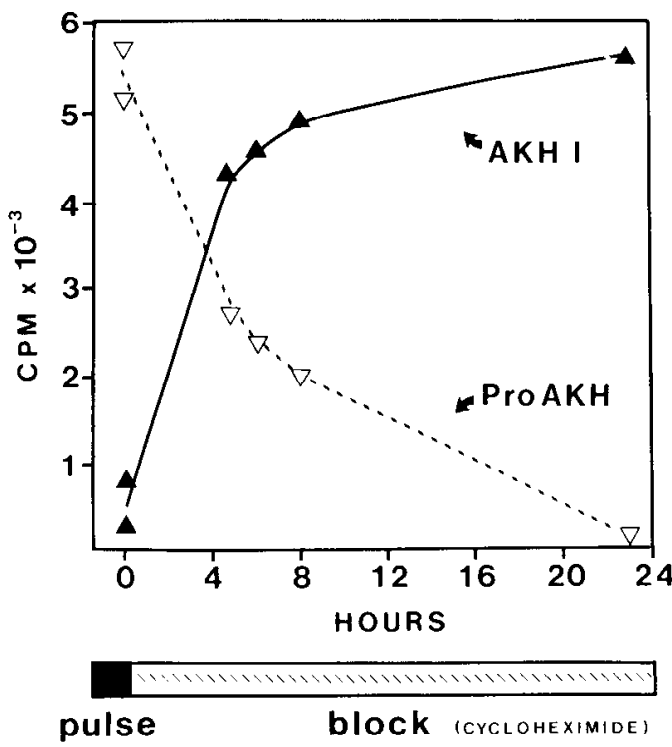

Figure 8. Time course of transfer of counts from labeled proAKH into AKH $I$ in a pulse-translation block experiment. Following a standard $1.5 \mathrm{hr}$ incubation in the presence of ${ }^{3} \mathrm{H}$-tryptophan (pulse), the glands were placed in medium containing $50 \mu \mathrm{g} / \mathrm{ml}$ cycloheximide (block). At 4 different times during the block, 3 glands were removed and chromatographed. Label in proAKH and AKH I was measured. Compare to Figure 7 and refer to text for further discussion.

I is provided by immunochemical experiments, described below. Also, we show that proAKH is not homogenous, but consists of 2 tritium-labeled polypeptides of about the same molecular weight, which comigrate on the HPLC (TSK 2000) size-exclusion column.

\section{Further purification and immunoreactivity of proAKH}

Using a rabbit antiserum to a synthetic peptide (Lys-Tyr-LeuAsn-Phe-Thr-Pro-Asn-Trp-Gly-Thr-OH; see Materials and Methods), we showed that proAKH contains an epitope recognized by this antiserum. We purified proAKH by TSK gelpermeation chromatography. The eluate from the column was collected in a tube from which the solvents were removed. The dried material was then dissolved in the RIA buffer (see Materials and Methods). Different CC equivalents of the purified proAKH (from 0.008 to $20 \mathrm{CC}$ equivalents) were assayed by RIA for AKH immunoreaclivity. The labeled tracer was the synthetic analog used for immunization. Figure 9 shows that proAKH was able to displace labeled tracer from antibody binding. Both proAKH and AKH I produced nearly parallel displacement curves. This indicated that the epitopes recognized on both molecules are similar.

Gel-permeation chromatography is not a high-resolution method, so we could not be sure that proAKH represented a single compound. We therefore attempted to fractionate ${ }^{3} \mathrm{H}-$ labeled proAKH. Glands were incubated with ${ }^{3} \mathrm{H}$-tryptophan and the labeled proAKH was collected from the eluant of the TSK 2000 column. The proAKH-containing fraction was applied to a large-pore $(300 \AA) \mathrm{C}_{8}$ column. ProAKH was now resolved into 2 primary compounds, P1 and P2 (Figs. 10, 11), both of which incorporated ${ }^{3} \mathrm{H}$-tryptophan. That P1 and P2 constitute proAKH is shown in Figure 10, which illustrates an experiment in which $\mathrm{CC}$ were incubated for $6 \mathrm{hr}$ with tritiated tryptophan and subsequently chromatographed by gel-permea- 


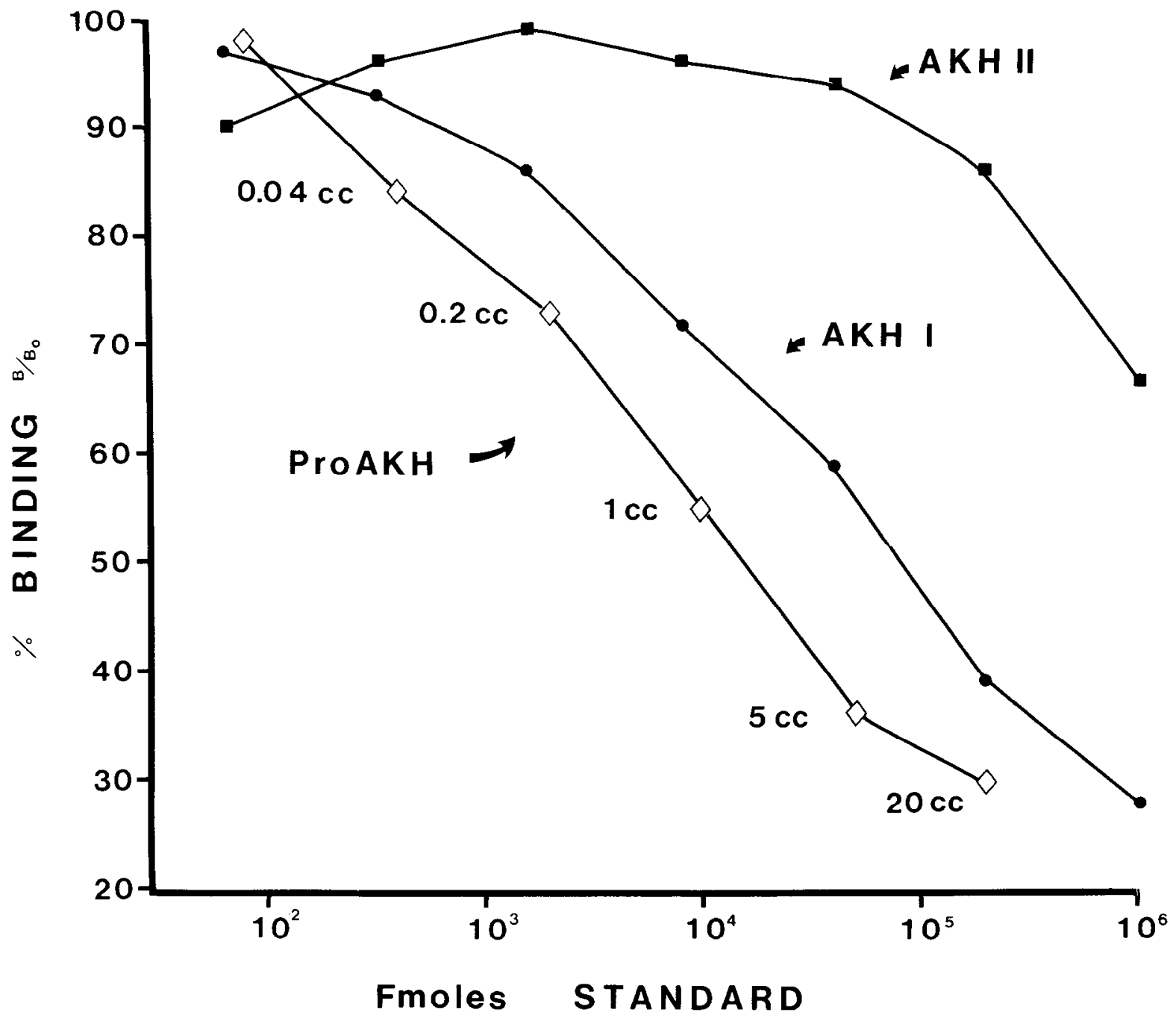

Figure 9. Detection of proAKH and AKH I by radioimmunoassay. See Materials and Methods for a description of the antiserum and immunogen. The proAKH was purified by TSK 2000 SW chromatography, dried, and then diluted in the RIA buffer to the CC equivalents shown on the graph. Synthetic standards of AKH I and II were used to generate the AKH I and II displacement curves. The parallelism of the curves for proAKH and AKH I suggests that similar epitopes are recognized. As expected from the structure of the immunogen, AKH II is only feebly recognized. The horizontal scale indicates the amounts of synthetic AKH I or II that produce a given displacement. The vertical scale indicates the proportion of the maximal binding $\left(B_{0}\right.$, tracer binding without nonlabeled antigen) achieved for each standard or experimental sample. Notice that the horizontal position of the $\mathrm{CC}$ displacement curve is arbitrary.

tion (see center, Fig. 10). The peak of radiolabel, proAKH, was then collected and equal aliquots of it were rechromatographed by both gel-permeation (left, Fig. 10) and reverse-phase (right, Fig. 10). Although fluorescence and optical density peaks other than P1 and P2 were resolved by the reverse-phase chromatography of purified proAKH, the only major peaks with radiolabel were P1 and P2 (right, Fig. 10). These are also the major fluorescence peaks. Thus, biosynthetically labeled proAKH consists of 2 tritiated compounds, $\mathrm{P} 1$ and $\mathrm{P} 2$, which can be separated by reverse-phase chromatography. Certainly some of the material associated with the tritiated proAKH peak on the TSK 2000 column is not itself radiolabeled during incubation with ${ }^{3} \mathrm{H}$-tryptophan. The major nonlabeled peak (77 a.u. in Fig. 10 ), separated by $\mathrm{C}_{8}$ reverse-phase chromatography, can be seen at the beginning of the chromatogram shown on the top right of Figure 10. When the P1 and P2 compounds are collected and rechromatographed on the TSK 2000 column, they comigrate with proAKH. When rechromatographed together on the TSK
2000 column, P1 and P2 are not resolved but run as a single peak (data not shown).

The homogeneity and AKH-relatedness of both radiolabeled and unlabeled P1 and P2 are supported by the following chromatographic and immunochemical observations. In the $\mathrm{CC}$ of adult animals there is always more ${ }^{3} \mathrm{H}$-tryptophan incorporated into P1 than P2, and more fluorescence and optical density associated with the former. This can be seen in both Figures 10 and 11. The typical adult incorporation ratio for $\mathrm{P} 1$ and $\mathrm{P} 2$ is about 0.5 . This is the same as the ratio of the peaks associated with each compound in Figures 10 and 11, for example. This ratio changes systematically during postembryonic development, and the change is highly correlated with a concomitant change in the ratio of the $2 \mathrm{AKH}$ peptides (S. Hekimi and M. O'Shea, unpublished observations).

That $\mathrm{P} 1$ and $\mathrm{P} 2$ are homogenous compounds is supported by reverse-phase chromatographic evidence, which shows single, well-defined peaks for each, using a variety of conditions. For 


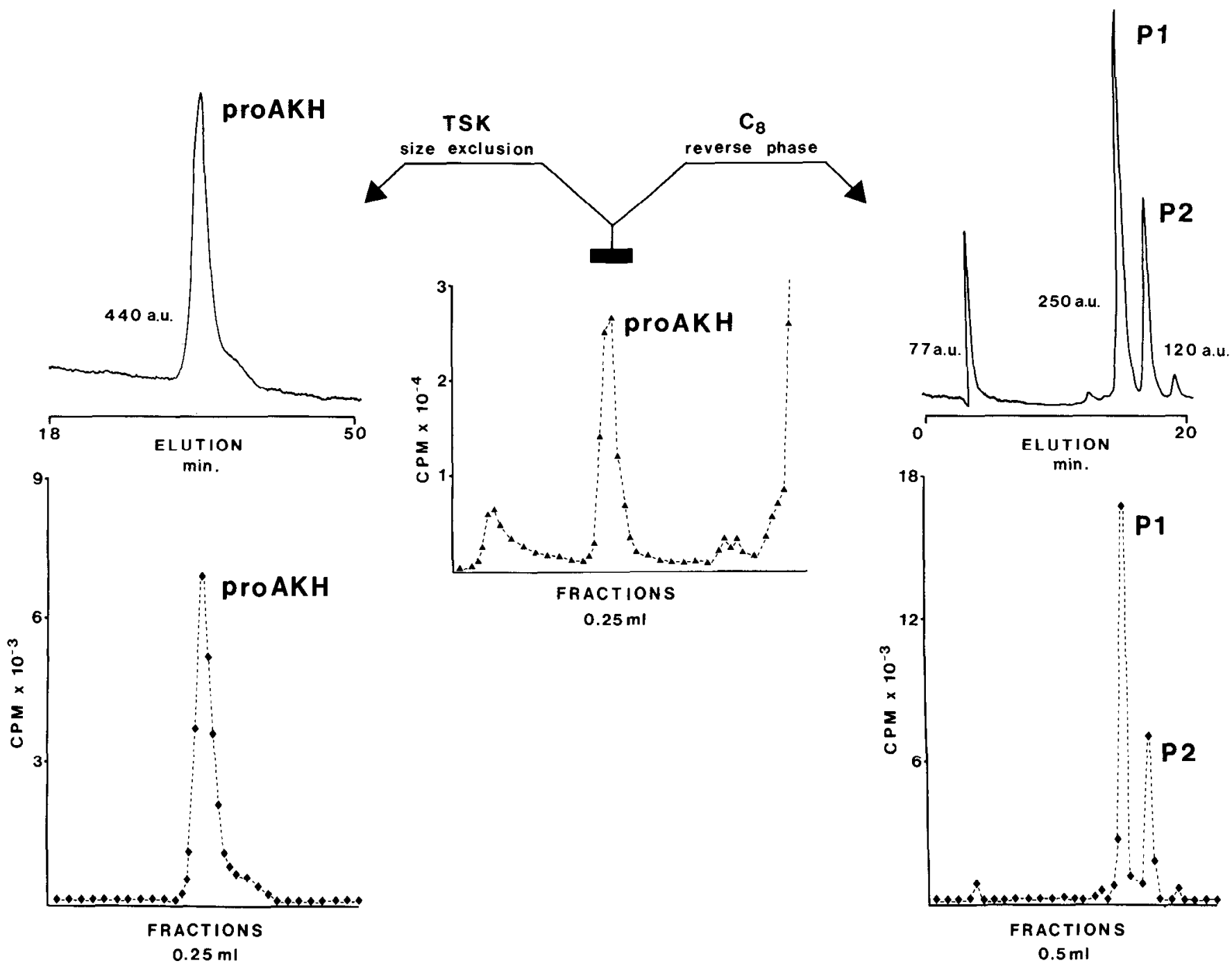

Figure 10. Further fractionation of proAKH. Forty $\mathrm{CC}$ were incubated for $6 \mathrm{hr}$ with ${ }^{3} \mathrm{H}$-tryptophan, extracted as usual, and chromatographed on the TSK $2000 \mathrm{SW}$ column. Fractions of $0.25 \mathrm{ml}$ were collected, from which a $25 \mu \mathrm{l}$ aliquot was counted for tritium. The result of this experiment is shown in the central graph of the figure. The prominent peak of label near the center of the trace was defined as proAKH by our experiments. The 6 fractions representing proAKH were pooled as indicated by the bar above the peak. Half of this pool was run back on the TSK column (left side of figure). The other half of the pool was passed on a $C_{8} 300 \AA$ reverse-phase column (right side of figure). The upper part of the right and left sides of the figure shows the fluorometric trace at an excitation wavelength of $276 \mathrm{~nm}$. The numbers associated with the peaks are arbitrary units (a.u.) representing the peak areas. Integration was performed by a Varian digital recorder. The lower part of the right and left sides of the figure shows the tritium counts associated with the fluorometric trace above. For further discussion, see text.

example, we used isocratic and several different gradients of TFA and acetonitrile, and a different eluting buffer $\left(\mathrm{NaPO}_{4}\right)$ and $\mathrm{pH}(7.0)$. None of the chromatographic conditions we employed have allowed us to separate the radiolabel from the optical density or fluorescence peaks. Furthermore, the $\mathrm{P} 1$ and $\mathrm{P} 2$ peaks described either by label or optical density are virtually superimposable (Fig. 11). Figure 11 shows a chromatogram of a crude extract of the $\mathrm{CC}$, and the high degree of purity that can be achieved in one step of $\mathrm{C}_{8}$ reverse-phase chromatography. Finally, with regard to purity, when P1 and P2 (isolated by 2 steps of $\mathrm{C}_{8}$ chromatography) are subjected to Edman sequencing, they yield a single amino acid per cycle of degradation.

Both $\mathrm{P} 1$ and $\mathrm{P} 2$ are recognized by the serum raised against an AKH analog (see Materials and Methods). This is illustrated in Figure 12, which shows the results of an RIA applied to fractions taken from a reverse-phase chromatogram of CC previously incubated with tritiated tryptophan. The distribution of label incorporated into $\mathbf{P} 1$ and $\mathbf{P} 2$ shows the characteristic difference (more in P1 than P2). This is also reflected in the relative $\mathrm{AKH}$-like immunoreactivity found in the fractions spanning the $\mathrm{P} 1$ and $\mathrm{P} 2$ peaks. Figure 12 presents the immunoreactivity expressed as percentage displacement. This can be transformed into moles of AKH-like immunoreactivity by means of a comparison with a standard AKH RIA curve. This calculation was performed by integrating the immunoreactive fractions 35-41 for P1 and 45-51 for P2. The P1 peak in Figure 12 contains about $12 \mathrm{pmol}$ of AKH-like immunoreactivity per $\mathrm{CC}$ and the P2 peak 4 pmol of AKH-like immunoreactivity. Since we do not know how the AKH peptides are distributed between P1 and $\mathrm{P} 2$ (see Discussion), there is no reason to expect that the immunoreactive ratio should be the same as the ratio of the 2 compounds detected by optical density, fluorescence, or label. Moreover, the interpretation of the detection of the $\mathrm{C}_{8}$ eluant by RIA is complicated by the fact that the antiserum recognizes 
Figure 11. Comparison of tritium incorporation and optical density associated with $\mathrm{P} 1$ and $\mathrm{P} 2\left(\mathrm{C}_{8}\right.$ reverse-phase chromatography). The fractions shown on the left graph were collected from the eluant profile shown on the right. Notice that the peak shapes and relative proportions are similar. This suggests that the optical density peaks and the peaks of label are produced by the same compounds.

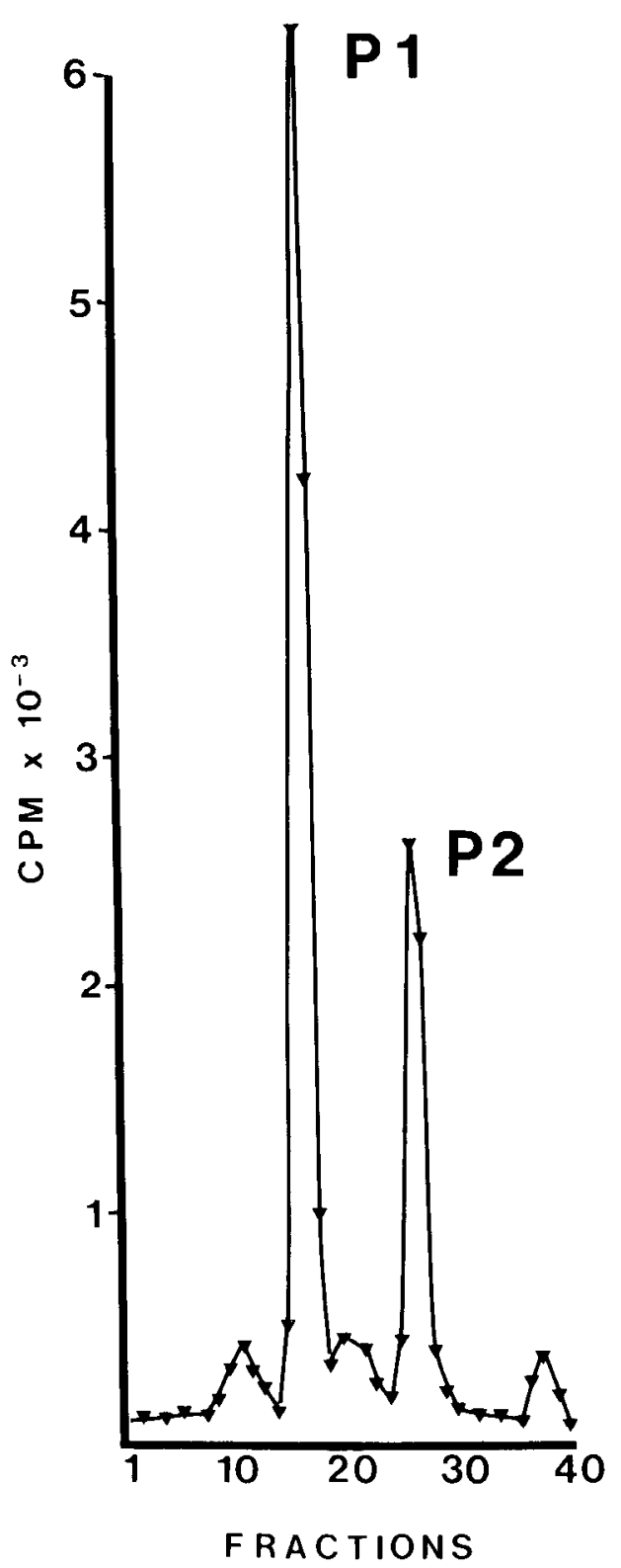

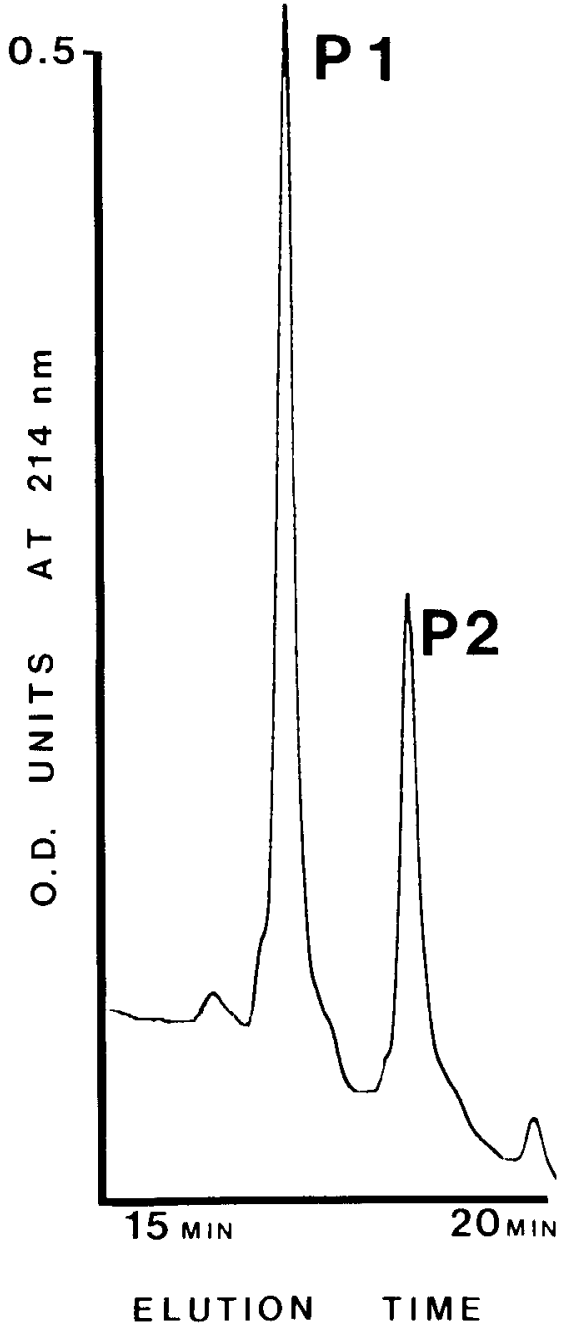

both AKH I and II, but has an aftinity an order of magnitude less for AKH II than for AKH I (see Fig. 9). In spite of these difficulties of quantification, it is clear that both P1 and P2 are recognized by the $\mathrm{AKH}$ antiserum.

\section{Discussion}

We have shown that the CC of the locust are the sites of synthesis of 2 major neuropeptides with adipokinetic activity, AKH I and AKH II. We have also shown that both are made in relatively large amounts by the intrinsic cells of the glandular lobes. We believe that 2 polypeptides, $\mathrm{Pl}$ and $\mathrm{P} 2$, participate in AKH I synthesis. This conclusion is supported by the observation that neither one alone can account quantitatively for the transfer of label to AKH I during pulse-chase or pulse-translation block experiments.

Several matters concerning P1, P2, and the biosynthesis of AKH I and II remain unresolved by our experiments. For example, we cannot be sure about the identity of the antecedent or antecedents of AKH II. It is possible that $\mathrm{Pl}$ and/or $\mathrm{P} 2$ are also AKH II precursors; this is not excluded by our results. Because, however, the rate of AKII II synthesis is only a small fraction of that of $\mathrm{AKH}$ I synthesis, we cannot be sure that there is not a precursor other than $\mathrm{P} 1$ or $\mathrm{P} 2$ that would be present and synthesized at low levels, accounting for AKH II synthesis.

Another unanswered question is whether P1 and $\mathrm{P} 2$ are products of independent translation. One of them could be a processing product of the other, perhaps representing the first step in the processing of the precursor. Indirect evidence suggests, however, that this is not the case, and that $\mathrm{P} 1$ and $\mathrm{P} 2$ are different translation products. This argument is based on the observation that during continuous incubation, the amount of label in proAKH $(\mathrm{P} 1+\mathrm{P} 2)$ reaches steady state in approximately $5 \mathrm{hr}$ (Fig. 5). Therefore, if $\mathrm{P} 1$ is derived from P2, or the reverse, an equilibrium between them cannot be reached before this time. In fact, our observations suggest that the relative proportions of P1 and P2 are established rapidly: At the earliest time at 


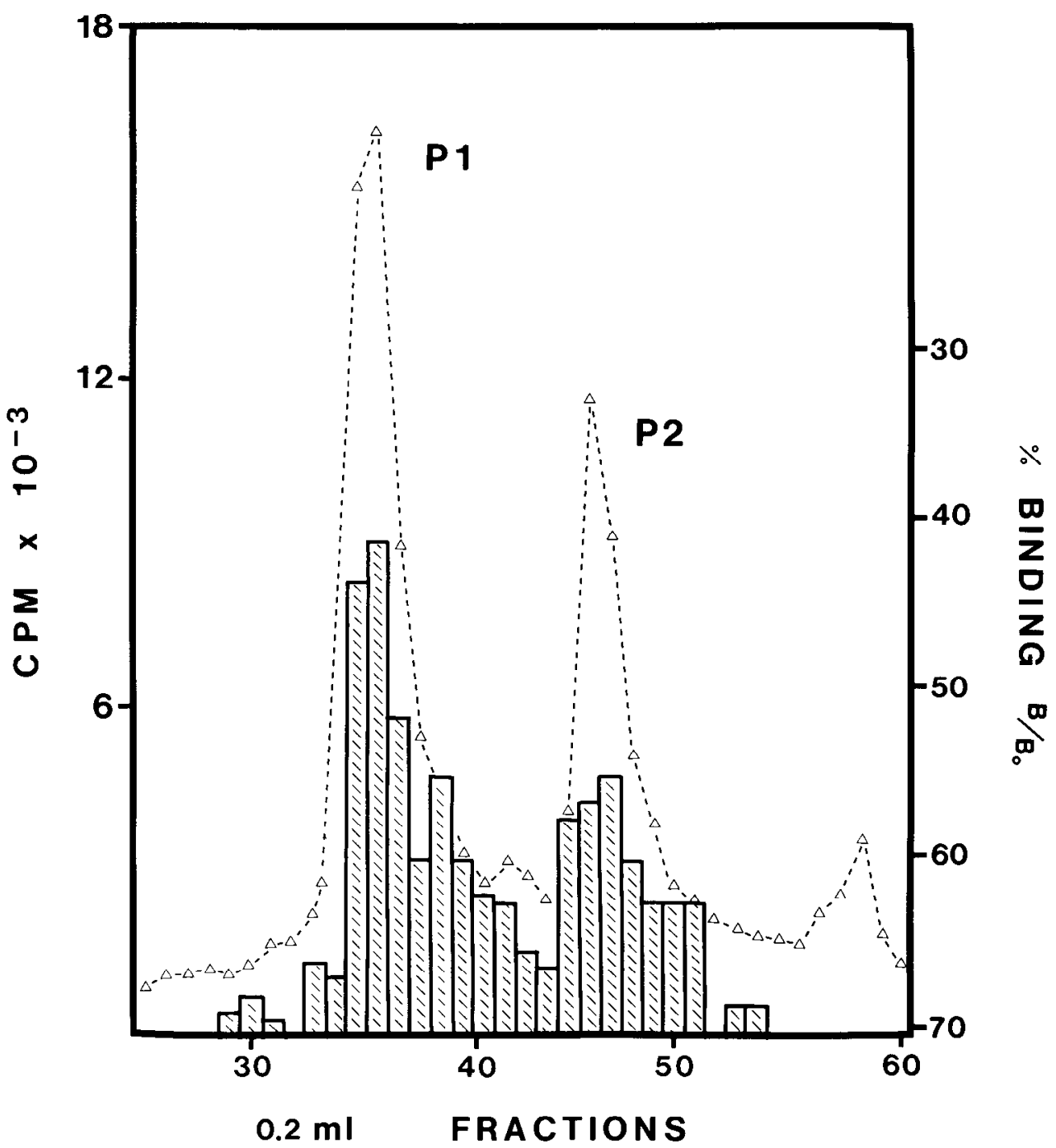

Figure 12. AKH immunoreactivity of $\mathrm{P} 1$ and $\mathrm{P} 2\left(\mathrm{C}_{8}\right.$ reverse-phase chromatography). A small aliquot of each fraction was counted for $\beta$-decays, and the remainder of each fraction was tested by RIA for AKH-like immunoreactivity. The hatched bars represent displacement of tracer from maximal binding $\left(B_{0}\right)$ achieved for every fraction. See also Figure 9. which we can detect radiolabeled $\mathrm{P} 1$ and $\mathrm{P} 2$, their typical relative proportion is already established (see, for example, Fig. 12).

The intrinsic cells of the glandular lobe synthesize both AKH I and II. Though these cells appear to be histologically homogenous (Fig. 1), we do not know whether they are homogenous with respect to peptide synthesis. Several possibilities exist. For example, all cells may make both peptides, or there may be separate populations of cells specialized with respect to peptide synthesis. It is possible that 2 such populations exist, one making AKH I, the other AKH II. We cannot exclude the possibility, however, that all cells make AKH I and a subpopulation makes both peptides. Some immunocytochemical evidence supports a nonhomogenous cell population hypothesis. For example, Schooneveld et al. (1986) have shown immunocytochemical evidence suggesting that a small subpopulation of cells (5-10\%) does not contain AKH I. This subpopulation is stained by an antiserum that recognizes both AKH I and II, but not by an AKH I-specific antiserum. In spite of this evidence, we consider the cellular basis for the difference between AKH I and AKH II synthesis rates to be unresolved.

Relevant to this discussion, of course, is the question of the cellular distribution of $\mathrm{P} 1$ and $\mathrm{P} 2$, which we believe to be dif- ferent translation products, which therefore could be found in different cell populations.

Many of the questions discussed above will be resolved by obtaining the complete primary structures of P1 and P2. Gasphase sequencing may allow us to achieve this because it can now be performed on subnanomolar amounts of peptide. We believe that the $\mathrm{CC}$ of locusts store unusually large quantities of $\Lambda \mathrm{KH}$ precursor (see below), and that complete sequencing of $\mathrm{P} 1$ and $\mathrm{P} 2$ can be expected. As we show in Figure 11, radiolabeled P1 and P2 comigrate with 2 major peaks of $214 \mathrm{~nm}$ optical density. The OD peaks appear to be uncontaminated; moreover, their relative surface areas are in the same proportion as the proportion of isotope in the labeled compounds. We conclude, therefore, that the OD peaks are produced by the 2 precursors $\mathrm{P} 1$ and $\mathrm{P} 2$. On the basis of the relative ODs of precursor and peptide, and from the immunoreactivity of P1 and $P 2$, we can calculate that a single CC, may contain from 10 to 100 pmol of precursor. Thus both P1 and P2 can be generated in sufficient quantities for sequencing by dissecting and pooling the $\mathrm{CC}$ of only a few hundred animals. This work is currently in progress.

The degree of reproducibility of our in vitro labeling experiments is also encouraging. We hope the system will allow us to 
study the influence of behavioral or pharmacological variables. For instance, the release of adipokinetic hormone is activated a few minutes after the initiation of flight. It is not yet known whether flight behavior or peptide release is associated with a regulation of peptide synthesis, but the system described here may allow us to address this question directly. It may also allow us to investigate the effects of substances that might be expected to regulate synthesis. One such compound is octopamine, which is known to cause the release of adipokinetic hormone, and which may be the factor that triggers $\mathrm{AKH}$ release after the initiation of flight (Orchard and Loughton, 1981).

Note added in proof: Recent experiments show that some of the OD shown comigrating with labeled P1 and P2 in Figure 11 is produced by high molecular weight fragments of the precursors.

\section{References}

Carlsen, J., W. S. Herman, M. Christensen, and L. Josefsson (1979) Chararacterization of a second peptide with adipokinetic and red pigment-concentrating activity from the locust corpora cardiaca. Insect Biochem. 9: 497-501.
Douglass, J., O. Civelli, and E. Herbert (1984) Polyprotein gene expression: Generation of diversity of neuroendocrine peptides. Annu. Rev. Binchem. 53: 665-715.

Mayer, R. J., and D. J. Candy (1969) Control of hemolymph lipid concentration during locust flight: An adipokinetic hormone from the corpora cardiaca. J. Insect Physiol. 25: 169-181.

Orchard, I., and B. G. Loughton (1981) Is octopamine a transmitter mediating hormone release in insects? J. Neurobiol. 12: 143-153.

O'Shea, M., and M. Schaffer (1985) Neuropeptides in invertebrates. Annu. Rev. Neurosci. 8: 171-198.

Schooneveld, H., H. M. Romberg-Privee, and J. A. Veenstra (1986) Immunocytochemical differentiation between adipokinetic hormone (AKH)-like peptides in neurons and glandular cells in the corpus cardicum of Locusta migratoria and Periplaneta americana with C-terminal and N-terminal specific antisera to AKH. Cell Tissue Res. 243: 9-14.

Siegert, K. J., and W. Mordue (1986) Quantification of adipokinetic hormones I and II in the corpora cardiaca of Schistocerca gregaria and Locusta migratoria. Comp. Biochem. Physiol. 84A: 279-284.

Siegert, K. J., P. Morgan, and W. Mordue (1985) Primary structures of locust adipokinetic hormones II. Hoppe-Seylers Z. Physiol. Chem. 366: 723-727.

Stone, J. V., W. Mordue, K. E. Batley, and H. R. Morris (1976) Structure of locust adipokinetic hormone that regulates lipid utilisation during flight. Nature 263: 207-211. 